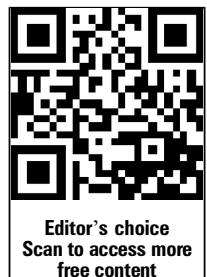

free content
${ }^{1}$ Section of Social Medicine, Department of Public Health, University of Copenhagen, Copenhagen K, Denmark ${ }^{2}$ Division of Epidemiology, Institute of Public Health, University of Southern Denmark, Odense, Denmark ${ }^{3}$ Department of Occupational Medicine, Danish Ramazzini Center, Herning Hospital, Herning, Denmark

${ }^{4}$ Department of Occupational and Environmental Medicine, Bispebjerg University Hospital, Copenhagen NV, Denmark

\section{Correspondence to} Professor Anne-Marie Nybo Andersen, Section of Social Medicine, Department of Public Health, University of Copenhagen, Oster

Farimagsgade 5, Box 2099, Copenhagen K DK-1014, Denmark;

amny@sund.ku.dk

Published Online First 9 July 2013

\title{
Occupational lifting of heavy loads and preterm birth: a study within the Danish National Birth Cohort
}

\author{
Stine Bjerrum Runge, ${ }^{1}$ Jacob Krabbe Pedersen, ${ }^{2}$ Susanne Wulff Svendsen, ${ }^{3}$ \\ Mette Juhl, ${ }^{1}$ Jens Peter Bonde, ${ }^{4}$ Anne-Marie Nybo Andersen ${ }^{1}$
}

\begin{abstract}
Objectives To examine the association between occupational lifting during pregnancy and preterm birth. The risk of preterm birth was estimated for total burden lifted per day and number of medium and heavy loads lifted per day.

Methods In a study population of 62803 pregnant women enrolled to the Danish National Birth Cohort from 1996 to 2002, the association between selfreported occupational lifting in the first part of pregnancy and preterm birth was analysed using logistic regression models with adjustment for age, parity, cervical cone biopsy, assisted reproduction and smoking. Associations between lifting and extremely (before 28 weeks), very (28-32 weeks) and moderately (33-37 weeks) preterm birth were analysed using Cox regression models.
\end{abstract}

Results We found a dose-response relation between total daily burden lifted and preterm birth with an OR of 1.50 (95\% Cl 1.03 to 2.19) with loads over $1000 \mathrm{~kg} /$ day. No threshold value was found. The associations were strongest for extremely and very preterm birth with HRs (95\% Cls) of 4.3 (1.4 to 13.8) and $1.7(0.7$ to 4.0$)$, respectively. Lifting heavy loads (>20 kg) more than10 times/day was associated with preterm birth up to an OR of $2.03(95 \% \mathrm{Cl} 1.14$ to 3.62). Conclusion In a society with social welfare and a highly regulated working environment, occupational lifting was associated with an increased risk of preterm birth.

\section{INTRODUCTION}

According to antenatal guidelines in, for example, Denmark, Norway and the UK, pregnant women should not carry heavy loads. ${ }^{1-3}$ These guidelines are formulated in broad terms and provide no information on a recommended maximum weight or maximum number of loads lifted per day during pregnancy. Previous Danish guidelines from 2002 suggested a maximum load of $12 \mathrm{~kg}$ and a maximum cumulative daily load of $1000 \mathrm{~kg}$, with halved limits in the third trimester, ${ }^{4}$ whereas recent guidelines from 2009 concentrate on lifting only as part of combined physical strain. ${ }^{1}$ At the same time, daily physical exercise in pregnancy is encouraged by health authorities in many countries. ${ }^{15} 6$

Even though the association between occupational lifting on the risk of preterm birth has been evaluated previously, ${ }^{7-13}$ the picture is inconsistent. A review including 12 studies found a more than moderate adverse effect of lifting unlikely. ${ }^{9}$ Relative

\section{What this paper adds}

- Antenatal counselling to pregnant women includes a general warning against lifting heavy loads during pregnancy, whereas recent guidelines from the Danish Working Environment Authority have omitted the previous explicit warning against lifting loads more than $12 \mathrm{~kg}$ each and a maximum cumulative daily load of $1000 \mathrm{~kg}$.

- A review of the literature concludes that a more than moderate adverse effect of lifting during pregnancy is unlikely but the findings are inconsistent.

- Using data from the Danish National Birth Cohort we were able to estimate the risk of preterm birth according to occupational lifting of burdens among more than 62000 women who were occupationally active during pregnancy and for whom self-reported data on work-related lifting were available.

- Heavy lifting was associated with an increased risk of preterm birth as a dose-response relation between cumulative daily load lifted and preterm birth risk was demonstrated. The associations were strongest for extremely and very preterm birth.

- As preterm birth is an adverse reproductive outcome with short-term and long-term consequences for the offspring, we think that heavy occupational lifting should be avoided during pregnancy.

risks (RRs) varied between 0.81 and 1.49 and were not statistically significant. If there is an effect of lifting heavy loads during pregnancy this may well differ according to the gestational age at birth. Recently, two studies were published from the Generation R birth cohort and the Southampton Women's study, showing no association between heavy lifting and preterm birth. ${ }^{14} 15$

The pathogenesis of preterm birth is not well understood. ${ }^{16}$ It has been suggested that release of catecholamines into the blood stream in response to physical or psychological stress could lead to uterine irritability, cervical changes and preterm birth. $^{8}{ }^{12}$ A weakened immune system due to stress 
could also lead to infections that trigger preterm birth. ${ }^{8} 17$ Further, changes in plasma levels of corticotrophin releasing factor and its binding protein may mediate an effect of stress on preterm birth. ${ }^{12}{ }^{17-19}$ Other hypotheses suggest that reduced uterine blood flow due to physical exercise initiates labour, ${ }^{7} 20$ or that heavy lifting increases abdominal pressure, which may provoke uterine contractions. ${ }^{7}$

The aim of this study was to examine the association between occupational lifting of heavy loads and preterm birth using data from a large, population-based cohort study. We investigated the association between the total daily load lifted and the number of loads lifted per day, and preterm birth. We also examined a possible time-dependant association.

\section{METHODS}

\section{Study population}

We used data from the Danish National Birth Cohort (DNBC), a nationwide follow-up study of pregnant women and their offspring with recruitment from 1996 to 2002. Recruitment took place with the general practitioner at the first pregnancy visit. Candidates were women who intended to carry their pregnancy to term and who spoke Danish well enough to participate in telephone interviews. About $60 \%$ of all pregnant women were invited and of these around $60 \%$ participated, resulting in a participation rate of about $35 \%$ of all pregnancies during the period. This specific study was based on the 90165 pregnancies with information from the first telephone interview in pregnancy, which was scheduled to take place in gestational week 12-16. More information about the DNBC can be found elsewhere. $^{21}$

To avoid non-independent observations, we only included the first pregnancy of each woman in the cohort $(n=81724)$, in case of more than one pregnancy of the same women in the cohort. Only live born singletons $(n=78$ 637) born after 22 completed weeks $(n=78635)$ and with a first pregnancy interview before 37 completed weeks $(n=78615)$ were included. Further, only women who had been working within 3 months before the interview $(n=64349)$ and for whom we had complete information on exposure variables and other included covariates $(\mathrm{N}=62803)$ were included.

\section{Measurement of exposure}

We used self-reported data on occupational lifting of heavy loads. In the interview, the women were asked:

1. In your job, do you daily lift more than $20 \mathrm{~kg}$ at a time (approximately like a crate of beer)?

2. How many times a day, do you lift more than $20 \mathrm{~kg}$ ? (if 'yes' to the above)

3. In your job, do you daily lift between 11 and $20 \mathrm{~kg}$ at a time (less than a crate of beer and more than a bucket of water)?

4. How many times a day do you lift between 11 and $20 \mathrm{~kg}$ ? (if 'yes' to the above)

A crate of beer is a solid plastic crate holding 30 beers in glass bottles. It weighs about $22 \mathrm{~kg}$ and is a generally well known item in Denmark. A version of the full questionnaire is available online (http://www.bsmb.dk). Women who reported 'less than once daily' in questions 2 or 4 were regarded non-lifters. In the following, $11-20 \mathrm{~kg}$ is referred to as medium loads and $>20 \mathrm{~kg}$ as heavy loads.

Based on the above questions two measures of exposure were generated: total daily load lifted, calculated so lifting a burden between 11 and $20 \mathrm{~kg}$ counted for $15 \mathrm{~kg}$ and lifting a burden more than $20 \mathrm{~kg}$ counted for $22.5 \mathrm{~kg}$ and categorised as shown in table 1; and combined frequencies of lifting. The latter was based on a cross table with weight $(0,11-20 \mathrm{~kg},>20 \mathrm{~kg})$ and frequency $(0,1-10$ loads/day and $>10$ loads/day).

\section{Measurement of outcome}

Preterm birth was defined as a delivery after 22 and before 37 completed gestational weeks (after 153 and before 259 days) with further a priori defined categorisation into extremely (22-27 completed weeks), very (28-32 completed weeks) and moderately preterm birth (33-36 completed weeks). Information on gestational age at birth came from the Medical Birth Registry, which is part of the National Patient Registry in Denmark. The information on gestational age at birth stem from the midwives' clinical assessment at birth, based on second trimester ultrasound estimation and date of last menstrual period.

\section{Measurement of covariates}

We had information on maternal age at conception and parity from the National Patient Registry. We obtained self-reported information about smoking during pregnancy, pre-pregnancy body mass index (BMI), having ever had a cervical cone biopsy, and whether the pregnancy was a result of assisted reproductive technologies (ARTs) from the DNBC. Furthermore, we had selfreported information about psychological stress at work ('is your job psychologically demanding?'), weekly working hours, leisure time lifting of loads $>20 \mathrm{~kg}$ per day, predominant work posture, primigravidity and job titles (which were registered by the interviewer according to the Danish version of the International Standard Classification of Occupation).

\section{Statistical analysis}

Non-proportional hazards were found when we applied Cox regression to the whole time range of preterm births (2236 weeks). We therefore used logistic regression models to analyse the relation between lifting of heavy loads and overall preterm birth. Maternal age, parity, ever had a cervical cone biopsy, ART and smoking during pregnancy were included as potential confounders based on existing literature. For the remaining selected potential confounders (pre-pregnancy BMI, psychological stress at work, working hours and lifting $>20 \mathrm{~kg}$ outside work) we did backwards stepwise elimination as follows: if elimination changed one of the estimates more than $2 \%$, the covariate should be included in the final model. However, this was not the case for any of these covariates and consequently they were left out.

For the association between total daily load lifted and preterm birth we conducted a test for trend using likelihood ratios. A test for threshold, using a method described by Bonde $e t a l,{ }^{22}$ revealed no signs of such. In a sub-analysis we stratified the analyses according to whether the woman had a job title that most likely implied lifting live humans or not, that is, patients, older people, children etc. Job titles with lifting humans were defined as nurses and nursing assistants; physiotherapists; hospital porters; women working in day care, whether at home or in institutions; and women working in institutions for people with specific needs, nursing homes or with home care. Other sub-analyses were carried out, one including work posture in the model as an additional, potential confounder, one restricted to primigravid women, and two in which observations with missing data on lifting were assigned no lifting or lifting 201-500 kg per day. For the combined frequencies of medium and heavy load lifting we tested for interaction between lifting the two weight loads. To examine potential timedependent associations between total daily load lifted and preterm birth, we estimated the association between lifting and 
Table 1 Distribution (percentages) of maternal characteristics according to cumulative daily loads lifted at work

\begin{tabular}{|c|c|c|c|c|c|c|c|c|}
\hline & No lifting & $15-50 \mathrm{~kg}$ & $51-100$ kg & $101-200$ kg & $201-500 \mathrm{~kg}$ & $501-1000 \mathrm{~kg}$ & $>1000 \mathrm{~kg}$ & Total \\
\hline & $\mathrm{N}=46199$ & $\mathrm{~N}=5326$ & $N=3396$ & $\mathrm{~N}=3342$ & $\mathrm{~N}=3043$ & $\mathrm{~N}=1022$ & $\mathrm{~N}=475$ & $\mathrm{~N}=62803$ \\
\hline & 73.6 & 8.5 & 5.4 & 5.3 & 4.9 & 1.6 & 0.8 & 100.0 \\
\hline \multicolumn{9}{|l|}{ Maternal age } \\
\hline$<25$ years & 9.1 & 16.2 & 15.6 & 17.2 & 17.4 & 18.9 & 21.4 & 11.1 \\
\hline $25-29$ years & 40.8 & 43.6 & 44.3 & 42.7 & 40.7 & 42.8 & 39.5 & 41.3 \\
\hline $30-34$ years & 37.5 & 30.9 & 30.7 & 30.1 & 31.5 & 29.2 & 29.8 & 35.7 \\
\hline$>34$ years & 12.7 & 9.3 & 9.4 & 10.1 & 10.4 & 9.1 & 9.2 & 11.9 \\
\hline Missing & $\mathrm{N}=0$ & $\mathrm{~N}=0$ & $\mathrm{~N}=0$ & $\mathrm{~N}=0$ & $\mathrm{~N}=0$ & $\mathrm{~N}=0$ & $\mathrm{~N}=0$ & $\mathrm{~N}=0$ \\
\hline \multicolumn{9}{|l|}{ Parity } \\
\hline 0 & 52.7 & 52.6 & 48.7 & 44.7 & 41.1 & 40.5 & 39.7 & 51.2 \\
\hline$\geq 1$ & 47.3 & 47.4 & 51.3 & 55.3 & 59.0 & 59.5 & 60.3 & 48.8 \\
\hline Missing & $\mathrm{N}=23$ & $\mathrm{~N}=3$ & $\mathrm{~N}=1$ & $\mathrm{~N}=1$ & $\mathrm{~N}=0$ & $\mathrm{~N}=3$ & $\mathrm{~N}=0$ & $\mathrm{~N}=31$ \\
\hline \multicolumn{9}{|l|}{ Cervical cone biopsy } \\
\hline Yes & 2.0 & 1.7 & 1.9 & 1.7 & 1.9 & 2.1 & 2.5 & 1.9 \\
\hline No & 98.0 & 98.3 & 98.2 & 98.3 & 98.1 & 98.0 & 97.5 & 98.1 \\
\hline Missing & $\mathrm{N}=2$ & $\mathrm{~N}=1$ & $\mathrm{~N}=0$ & $\mathrm{~N}=1$ & $\mathrm{~N}=0$ & $\mathrm{~N}=0$ & $\mathrm{~N}=0$ & $\mathrm{~N}=4$ \\
\hline \multicolumn{9}{|l|}{ IVF/ICSI treatment } \\
\hline Yes & 2.6 & 1.9 & 1.2 & 1.6 & 1.9 & 2.0 & 2.9 & 2.4 \\
\hline No & 97.4 & 98.1 & 98.8 & 98.4 & 98.1 & 98.1 & 97.1 & 97.6 \\
\hline \multicolumn{9}{|c|}{ Smoking during pregnancy } \\
\hline Yes & 12.1 & 15.9 & 18.7 & 20.5 & 23.6 & 28.5 & 32.2 & 14.2 \\
\hline No & 87.9 & 84.1 & 81.3 & 79.5 & 76.4 & 71.5 & 67.8 & 85.8 \\
\hline Missing & $N=21$ & $\mathrm{~N}=4$ & $\mathrm{~N}=3$ & $\mathrm{~N}=0$ & $\mathrm{~N}=0$ & $\mathrm{~N}=0$ & $\mathrm{~N}=1$ & $\mathrm{~N}=29$ \\
\hline \multicolumn{9}{|l|}{ Pre-pregnancy BMI } \\
\hline$<18.5$ & 4.4 & 3.6 & 4.1 & 3.7 & 4.1 & 3.9 & 4.3 & 4.2 \\
\hline $18.5-<25$ & 70.7 & 65.9 & 64.6 & 63.2 & 59.8 & 57.9 & 54.4 & 68.7 \\
\hline $25-<30$ & 18.3 & 22.0 & 21.2 & 21.7 & 22.9 & 23.1 & 27.4 & 19.4 \\
\hline$\geq 30$ & 6.6 & 8.5 & 10.1 & 11.4 & 13. 2 & 15.1 & 14.0 & 7.7 \\
\hline Missing & $\mathrm{N}=740$ & $\mathrm{~N}=84$ & $\mathrm{~N}=43$ & $\mathrm{~N}=55$ & $\mathrm{~N}=48$ & $\mathrm{~N}=10$ & $\mathrm{~N}=5$ & $\mathrm{~N}=985$ \\
\hline \multicolumn{9}{|c|}{ Psychological stress at work } \\
\hline Often & 15.9 & 19.9 & 21.3 & 25.4 & 28.1 & 28.8 & 24.2 & 17.9 \\
\hline Now and then/rarely & 84.1 & 80.1 & 78.7 & 74.6 & 71.9 & 71.2 & 75.8 & 82.1 \\
\hline Missing & $\mathrm{N}=33$ & $\mathrm{~N}=3$ & $\mathrm{~N}=1$ & $\mathrm{~N}=2$ & $\mathrm{~N}=1$ & $\mathrm{~N}=0$ & $\mathrm{~N}=0$ & $\mathrm{~N}=40$ \\
\hline \multicolumn{9}{|l|}{ Work week } \\
\hline Part time & 21.0 & 30.1 & 31.4 & 31.3 & 29.0 & 27.6 & 16.6 & 23.4 \\
\hline Full time & 79.0 & 69.9 & 68.6 & 68.7 & 71.0 & 72.4 & 83.4 & 76.6 \\
\hline Missing & $\mathrm{N}=21$ & $\mathrm{~N}=3$ & $\mathrm{~N}=1$ & $\mathrm{~N}=0$ & $\mathrm{~N}=0$ & $\mathrm{~N}=0$ & $\mathrm{~N}=0$ & $\mathrm{~N}=25$ \\
\hline \multicolumn{9}{|c|}{ Lifting $>20 \mathrm{~kg}$ daily outside work } \\
\hline Yes & 5.5 & 9.0 & 8.9 & 10.1 & 11.6 & 12.5 & 12.2 & 6.7 \\
\hline No & 94.5 & 91.0 & 91.1 & 89.9 & 88.4 & 87.5 & 87.8 & 93.3 \\
\hline Missing & $\mathrm{N}=41$ & $\mathrm{~N}=5$ & $\mathrm{~N}=1$ & $\mathrm{~N}=2$ & $\mathrm{~N}=4$ & $\mathrm{~N}=1$ & $\mathrm{~N}=0$ & $\mathrm{~N}=54$ \\
\hline \multicolumn{9}{|c|}{ Predominant work posture } \\
\hline Standing/walking & 17.8 & 45.5 & 53.3 & 57.9 & 58.1 & 68.2 & 76.1 & 27.4 \\
\hline Other & 82.2 & 54.5 & 46.7 & 42.1 & 42.0 & 31.8 & 24.0 & 72.6 \\
\hline Missing & $\mathrm{N}=28$ & $\mathrm{~N}=1$ & $\mathrm{~N}=0$ & $\mathrm{~N}=3$ & $\mathrm{~N}=4$ & $\mathrm{~N}=0$ & $\mathrm{~N}=0$ & $\mathrm{~N}=36$ \\
\hline \multicolumn{9}{|l|}{ Primigravidity } \\
\hline Yes & 59.8 & 59.2 & 63.7 & 65.8 & 69.8 & 70.7 & 72.1 & 61.0 \\
\hline No & 40.2 & 40.8 & 36.3 & 34.3 & 30.2 & 29.4 & 27.9 & 39.0 \\
\hline Missing & $\mathrm{N}=22$ & $\mathrm{~N}=3$ & $\mathrm{~N}=1$ & $\mathrm{~N}=1$ & $\mathrm{~N}=0$ & $\mathrm{~N}=3$ & $\mathrm{~N}=0$ & $\mathrm{~N}=30$ \\
\hline \multicolumn{9}{|l|}{ Gestational duration } \\
\hline $22-27+6$ weeks & 0.2 & 0.2 & 0.2 & 0.1 & 0.3 & 0.2 & 0.6 & 0.2 \\
\hline $28-32+6$ weeks & 0.6 & 0.5 & 0.5 & 0.9 & 0.9 & 0.8 & 1.0 & 0.7 \\
\hline $33-36+6$ weeks & 3.6 & 3.6 & 4.0 & 4.0 & 4.1 & 3.2 & 4.5 & 3.7 \\
\hline 37+weeks & 95.3 & 95.3 & 95.1 & 94.9 & 94.5 & 95.8 & 93.6 & 95.4 \\
\hline Missing & $\mathrm{N}=29$ & $\mathrm{~N}=1$ & $\mathrm{~N}=4$ & $\mathrm{~N}=4$ & $\mathrm{~N}=6$ & $\mathrm{~N}=1$ & $\mathrm{~N}=1$ & $\mathrm{~N}=46$ \\
\hline
\end{tabular}


extremely, very and moderately preterm birth, respectively, using Cox regression models. For extremely preterm birth, time at risk started from the day a woman completed gestational week 22 or by the time of the first pregnancy interview, whichever came last. Follow-up ended at birth or at completion of 27 weeks, whichever came first. A similar principle was used for very and moderately preterm birth using the corresponding gestational ages. Log-log plots were made to evaluate the proportional hazards assumption. All analyses were done with the STATA/IC V.10.1 software.

\section{RESULTS}

Overall 16604 women (26.4\%) carried heavy loads at work, and 475 women (2.9\%) lifted more than $1000 \mathrm{~kg}$ per day. Preterm birth occurred in 2870 pregnancies (4.6\%). Mean gestational age at birth was 280 days corresponding to 40.0 gestational weeks. Gestational age at interview varied between 6 and 40 weeks (mean 16) and did not differ between exposure groups. The majority of the women were interviewed before 22 weeks of gestation (86.5\%). From table 1 it follows that daily lifting was associated with young age, multigravidity, multiparity, smoking, pre-pregnancy BMI $>25$, psychological stress at work, part-time work, and leisure time lifting of heavy loads. Standing or walking at work was strongly associated with lifting. A minority of the women had been working within the past 3 months, but had stopped working before the time of the interview. As $1.3 \%$ of the total study population had a gestational age less than 3 months at the time of interview, this means that a very small proportion of the women included in our study might have stopped working already before they became pregnant. We found a strong correlation between being on sick leave at the time of interview and the cumulated daily load (increasing gradually from $8.6 \%$ of non-lifters to $43.2 \%$ of those lifting $>1000 \mathrm{~kg} /$ day) (data not shown).

Among the 1481 women who had missing data on occupational lifting of heavy loads, the most common job titles (shop, warehouse, factory and postal workers) corresponded mostly to women who reported lifting 201-500 kg per day (data not shown).

Figure 1 shows the association between the total daily loads lifted and preterm birth. A dose-response relation was found but with no apparent threshold value. The OR of preterm birth was 1.19 (95\% CI 1.02 to 1.40$)$ for women lifting $101-200 \mathrm{~kg}$

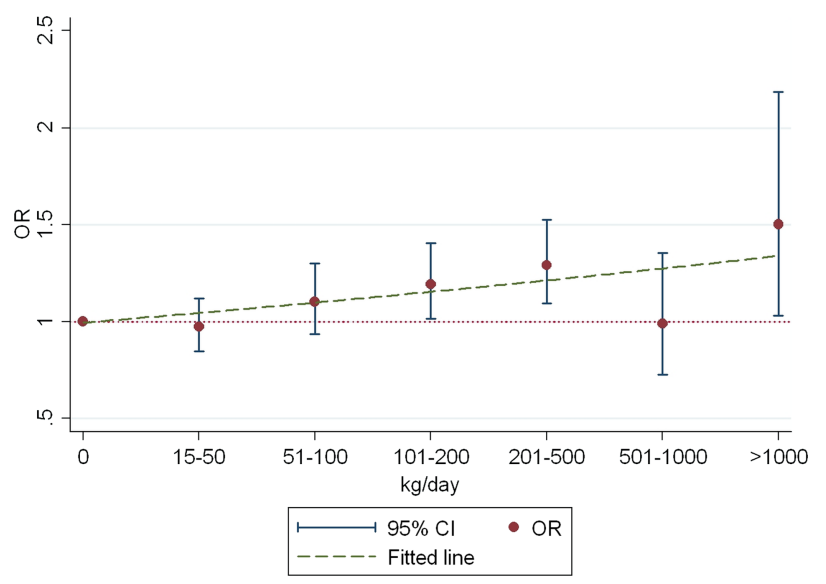

Figure 1 The relative risk of preterm birth (ORs with 95\% Cls) according to the cumulative daily load lifted at work in pregnancy. The Danish National Birth Cohort, 1996-2002. This figure is only reproduced in colour in the online version. per day with an increase up to 1.50 (95\% CI 1.03 to 2.19) among lifting $>1000 \mathrm{~kg}$ compared with non-lifters (figure 1). Test for trend was statistically significant $(p<0.001)$, even though lifting 501-1000 kg per day was not associated with preterm birth. Including maternal age, parity, cervical cone biopsy, assisted reproduction treatment and smoking in the model only had a marginal influence on the results.

When we repeated the analysis stratified for lifting humans, we found that having a job that implied lifting of heavy loads but most likely without lifting humans displayed higher ORs than the estimates for overall occupational lifting, while ORs were close to one for all levels of total daily burden lifted in women with jobs that most likely implied lifting humans (data no shown). The proportion of women on sick leave was similar in the two groups. The two additional sub-analyses, inclusion of work posture in the model and restriction to primigravid women, attenuated the associations.

The sensitivity analysis in which all women with missing data on lifting heavy loads during pregnancy were assigned to the non-lifting group revealed essentially unchanged estimates, while the estimates were slightly attenuated when the women with missing lifting data were assigned a total daily burden lifted of 201-500 kg/day (data not shown).

Table 2 shows ORs for the combined frequencies of occupational lifting. We found that medium and heavy weight loads were associated with preterm birth (table 2). The strongest association was seen when heavy loads were lifted more than 10 times per day. Adjustment did not change the estimates substantially. Test for interaction between lifting of medium and heavy loads was only just statistically significant $(p=0.03)$. Thus, interaction could not be ruled out, though no consistent pattern was apparent.

Table 3 shows HRs for the different degrees of preterm birth according to total daily load lifted. Occupational lifting was associated with preterm birth at all gestational ages, however at a statistically significant level in only few of the comparisons made. The strongest associations were in general seen for extremely preterm birth with a fourfold increased risk among women lifting more than $1000 \mathrm{~kg}$ per day. Within the highest groups of loads lifted per day, the HRs declined with increasing gestational age.

\section{DISCUSSION}

In this cohort study of preterm birth according to self-reported occupational lifting in 62803 pregnancies, a dose-response relation between total daily load lifted and preterm birth was found. Lifting loads of $11-20 \mathrm{~kg}$ and $>20 \mathrm{~kg}$ were associated with the outcome, but lifting loads $>20 \mathrm{~kg}$ more than 10 times daily had the strongest association with preterm birth. The associations persisted after control for possible confounders.

Previous studies on lifting and preterm birth differ largely with respect to method and measure of exposure. Mozurkewich et $a l^{12}$ did a meta-analysis of 21 studies of the association between physically demanding work (including heavy lifting) and preterm birth and found an OR of 1.22 (95\% CI 1.16 to 1.29 ). The analysis included case-control, cross-sectional and cohort studies and the results were consistent across all study designs and analysis methods. Bonzini et $a l^{9}$ did a review in 2008 and found 11 out of 12 RR estimates lower than 1.35 (95\% CI 0.81 to 1.49 ). No results showed statistical significance. Heavy lifting was usually defined as lifting more than $11-12 \mathrm{~kg}$, but the frequency varied a lot. Overall, the results of these two studies indicate an association of similar size as ours. 
Table 2 Adjusted* OR for preterm birth according to number of medium (11-20 kg) and heavy (>20 kg) loads lifted per day at work during pregnancy

\begin{tabular}{|c|c|c|c|c|c|c|}
\hline \multirow[b]{3}{*}{ Medium loads/dayt } & \multicolumn{6}{|c|}{ Heavy loads/day $\ddagger$} \\
\hline & \multicolumn{2}{|l|}{ None } & \multicolumn{2}{|l|}{$1-10$} & \multicolumn{2}{|l|}{$>10$} \\
\hline & Adjusted OR & $95 \% \mathrm{Cl}$ & Adjusted OR & $95 \% \mathrm{Cl}$ & Adjusted OR & $95 \% \mathrm{Cl}$ \\
\hline None & 1 & & 0.77 & (0.53 to 1.13 ) & 2.03 & (1.14 to 3.62$)$ \\
\hline $1-10$ & 1.07 & (0.95 to 1.20 ) & 1.19 & (1.04 to 1.36$)$ & 1.38 & (0.70 to 2.72$)$ \\
\hline$>10$ & 1.30 & (1.00 to 1.69 ) & 0.75 & (0.49 to 1.13 ) & 1.36 & (1.02 to 1.82 ) \\
\hline
\end{tabular}

62803 women in the Danish National Birth Cohort, 1996-2002.

${ }^{*}$ Adjusted for maternal age, parity, cervical cone biopsy, IVF/ICSI treatment and smoking.

tMean number of loads: 0, 4 and 35 loads/day, respectively.

¥Mean number of loads: 0,4 and 33 loads/day, respectively.

ICSI, intra-cytoplasmic sperm injection; IVF, in vitro fertilisation.

In one study, Pompeii et al ${ }^{13}$ did a cohort study of 1711 women and found a RR of 1.3 (95\% CI 0.8 to 2.1) for preterm birth with lifting in the second trimester. A wide definition of exposure was used (lifting more than $25 \mathrm{lbs}(\sim 11.34 \mathrm{~kg})$ over 13 times/week), which implies less power to reveal any negative effect of heavy lifting $(>20 \mathrm{~kg})$. Another large cohort study including 3389 women was done by Ahlborg et al. ${ }^{7}$ The study design was quite similar to ours, and lifting $>12 \mathrm{~kg}>50$ times/ week early in pregnancy yielded an OR of 1.29 (95\% CI 0.69 to 2.40). The most recent cohort studies, though, failed to find any relation. ${ }^{14}$ Both of these studies had their exposure assessment later in pregnancy, which could make them more susceptible to a 'healthy lifter' effect, a bias that may affect observational studies in settings in which pregnant women have any influence on their working conditions, and also ours.

Few previous works have examined any dose-response effect of lifting. Ahlborg et al divided exposure into four categories based on weight and frequency. No dose-response relation was found, as only maximum exposure ( $>12 \mathrm{~kg}>50$ times/week) was associated with preterm birth. ${ }^{7}$ This does not contradict our findings though, since the dose-response effect we found was mainly seen for groups with exposure above this limit. We did not find any previous works that evaluated the effect of two different weights comparable to our medium and heavy loads.

The present results are consistent with previous findings in that the hazardous effect of lifting seemed to be only moderate. Our cohort was much larger and some of our findings showed statistical significance. With a rare outcome, a quite rare exposure and only a moderate effect, a study population this size would be needed to obtain statistically significant results.

We found that the HR of preterm birth in relation to the cumulative daily load changed over gestational time and was largest for extremely preterm birth. Recently, we demonstrated that heavy lifting increases the risk of first trimester miscarriage. Whether the mechanism behind these findings is the same is speculative. ${ }^{23}$ Data from the DNBC has been used to assess the association between physical exercise and adverse pregnancy outcome. While leisure-time physical exercise was associated with miscarriage ${ }^{24}$ no such association was found to preterm birth. $^{25}$

Pompeii et $a l^{13}$ had information about lifting in the first and second trimesters and found that the proportion of women who reported lifting at work declined from the first to the second trimester, but in this study preterm birth was treated as a single entity.

This study was based on a large cohort of pregnant women, using prospectively collected data only and with complete follow-up. Participants in interview-based research like this may be expected to be a healthier selection from the general population. The present study was restricted to women who had been working within 3 months of the time of interview. This could be expected to yield an even healthier cohort, and indeed the proportion of preterm births was only $4.6 \%$ in this study compared with the overall proportion observed in Denmark from 1997 to $2003(5.2-6.2 \%) .^{26}$ The low participation rate may be a source of bias. In a validation study where risk estimates on

Table 3 Relative risk HR for three degrees of preterm birth according to the cumulative daily loads lifted at work in pregnancy

\begin{tabular}{|c|c|c|c|c|c|c|c|c|c|c|}
\hline \multirow[b]{3}{*}{ Loads per day } & \multirow[b]{3}{*}{$\mathrm{N}$} & \multicolumn{9}{|c|}{$\begin{array}{l}\text { Degree of preterm birth (full gestational weeks) } \\
\text { Number of events }\end{array}$} \\
\hline & & \multicolumn{3}{|c|}{ Extremely preterm (22-27) $\mathrm{N}=110$} & \multicolumn{3}{|c|}{ Very preterm (28-32) $\mathrm{N}=415$} & \multicolumn{3}{|c|}{ Moderately preterm (33-36) N=2.345 } \\
\hline & & Crude HR & Adjusted* HR & $(95 \% \mathrm{Cl})$ & Crude HR & Adjusted* HR & $(95 \% \mathrm{Cl})$ & Crude HR & Adjusted* HR & $(95 \% \mathrm{Cl})$ \\
\hline No lifting & 46.199 & 1 & 1 & & 1 & 1 & & 1 & 1 & \\
\hline $15-50 \mathrm{~kg}$ & 5.326 & 1.25 & 1.35 & (0.72 to 0.55$)$ & 0.82 & 0.82 & (0.56 to 1.21$)$ & 0.98 & 0.99 & (0.85 to 1.15$)$ \\
\hline $51-100 \mathrm{~kg}$ & 3.396 & 1.24 & 1.42 & (0.65 to 3.10$)$ & 0.82 & 0.84 & (0.52 to 1.36$)$ & 1.10 & 1.13 & (0.95 to 1.35$)$ \\
\hline $101-200 \mathrm{~kg}$ & 3.342 & 0.54 & 0.62 & (0.19 to 1.96$)$ & 1.35 & 1.41 & (0.96 to 2.07 ) & 1.12 & 1.17 & (0.98 to 1.40$)$ \\
\hline $201-500 \mathrm{~kg}$ & 3.043 & 1.59 & 1.84 & (0.88 to 3.83 ) & 1.54 & 1.61 & (1.10 to 2.36 ) & 1.14 & 1.20 & (1.00 to 1.44$)$ \\
\hline $501-1000 \mathrm{~kg}$ & 1.022 & 1.17 & 1.36 & (0.33 to 5.57 ) & 1.22 & 1.26 & (0.62 to 2.55 ) & 0.88 & 0.93 & (0.66 to 1.31 ) \\
\hline$>1000 \mathrm{~kg}$ & 475 & 3.86 & 4.32 & (1.35 to 13.82 ) & 1.65 & 1.65 & (0.68 to 4.00 ) & 1.28 & 1.34 & (0.88 to 2.05$)$ \\
\hline
\end{tabular}

62803 women in the Danish National Birth Cohort 1996-2002.

${ }^{*}$ Adjusted for maternal age, parity, cervical cone biopsy, IVF/ICSI treatment and smoking.

ICSI, intra-cytoplasmic sperm injection; IVF, in vitro fertilisation. 
three exposure-outcome associations based on data from the DNBC were compared with risk estimates calculated using total population-covering data it was demonstrated that the risks estimated were unbiased by the selection to the cohort. $^{27}$ Therefore, we find it likely that the results apply to women in general who work during the first trimester of pregnancy.

As a woman was interviewed only if she was still pregnant, some cases of extremely preterm birth may have been missed. The majority of women in the study were interviewed before gestational week 22, however, and since the time of interview did not depend on exposure or outcome, this would probably not bias the results.

The later outcomes depend on whether earlier outcomes occurred. For example, moderately preterm birth depends on extremely or very preterm birth not happening. This may have introduced bias; however, if any, most likely an underestimation of the HR.

Restricting the analysis to primigravidae showed smaller risk estimates. An explanation could be that women with previous preterm birth or complications in the existing pregnancy might overestimate their occupational lifting more than the general population while being more prone to preterm birth. Thereby, the effect of heavy lifting would be overestimated. However, previous negative experience with pregnancy could lead to selfselection into exposure groups with less or no exposure to lifting and vice versa. We would then expect a stronger association between lifting and preterm birth when restricting analysis to primigravidae. In general, ORs were closer to one and we believe that reverse causation due to experiences in previous pregnancies is a less likely source of bias.

The average values used for calculating the total daily load lifted were not validated and thus should be interpreted with caution. More than $20 \mathrm{~kg}$ was thought to equal on average $22.5 \mathrm{~kg}$ as we believed that few women lift more than $25 \mathrm{~kg}$ at work. As 11-20 kg was expected to equal $15 \mathrm{~kg}$, the relation between the two loads was estimated to be 3:2. Lifting loads of less than $11 \mathrm{~kg}$ was equal to no lifting and it seems reasonable that some lower thresholds exist under which the weight lifted is too small to exert any effect. The analysis of the combined frequencies of lifting medium and heavy loads showed that both weight categories should be included in the cumulative daily load. Further, according to their respective association with preterm birth, their estimated relation of 3:2 seemed reasonable.

Self-reported data on lifting generally provides only a crude assessment of exposure and this is a limitation of our study. Differential misclassification could occur if women who had a high a priori risk of preterm birth reported differently than those with no such risk. This is not very likely to occur in a cohort study. In a review on self-reported data about occupational lifting, Stock et $a l^{28}$ found that reproducibility was best using one or two weight classes. Compared with observations, validity was best for higher weights and improved when few categories of frequency were used. The continuous variable on frequency obtained through the questionnaire in DNBC was broken down into three rough categories before data analysis and overall the self-reported data used are thus expected to be of better quality.

It has anecdotally been mentioned that people having jobs that involve lifting humans tend to overestimate the load lifted. The use of mechanical lifting equipment, help from coworkers or the patient or child can make it hard to judge how much weight is actually lifted. We found that the association with preterm birth was stronger for women in jobs with no lifting of humans and that it seemed non-existent for women with jobs that most likely included lifting humans. This may be due to misclassification bias on self-reported data when lifting humans, which could lead to the failure to recognise an existing association. However, we have no reason to believe that occupational lifting of any actual weight affects the risk of preterm birth differently depending on the nature of the burden.

To get more precise exposure measures it would be necessary to make detailed observations of individual participants using accelerometers, activity logs etc. This is not manageable in a large cohort like this. It could be informative, however, to directly measure the loads lifted by a small selection of participants and compare these with their self-reported data to validate or adjust the self-reported data, if possible.

Including predominant work posture in the models could lead to over-adjustment since work posture and lifting were closely related in the data. Hence, this was done in a separate analysis, but the estimates did not change.

Overall 1481 women had missing data on exposure. Their job titles seemed to be most similar to the group lifting 201$500 \mathrm{~kg} /$ day. Therefore they were placed in this group in a sensitivity analysis and we found that estimates did not change much. So, missing information is unlikely to have biased our results.

There is general agreement that the timing of exposures during pregnancy is important. Lifting was evaluated only once early in pregnancy. This made prospective analysis possible for almost all preterm births in the cohort, but is also a major limitation of this study. With gestational time, the women and people around them will be increasingly aware of the pregnancy and may try to reduce physical strain. Some women had already stopped working before the interview and many of the remainder were expected to go on leave before week $37 .^{29}$ This likely exposure misclassification would lead to an underestimation of the effect of lifting. Since the proportion of women who had stopped working most probable increased by gestational age, the risk of very and moderately preterm birth may have been underestimated the most.

If the effect of lifting is short term, the time dependency of the effect of lifting could simply be due to the fact that the lifters gradually become non-lifters. That is, the effect on very and moderately preterm birth could have been underestimated.

Some women might have stopped working because of other known risk factors for preterm birth, factors that might be related to exposure. Any induced bias would lead to an underestimation of the risk of lifting. Further, the proportion of women who had stopped working by the time of interview increased considerably with the cumulative daily load lifted. This means that women with maximum exposure were exposed for a shorter time on average and hence the effect could have been underestimated. It seems that restrictive official guidelines at that time in Denmark were followed by many pregnant women and their general practitioners. Nonetheless an effect was found.

Even in a society with extensive regulations of the working environment, occupational lifting was associated with a moderately increased risk of preterm birth. Hazards at work are not well accepted, particularly not in pregnancy. In this study, a strong association was seen between lifting and extremely preterm birth. Birth in gestational week 22-27 is a serious outcome and prevention is highly important. However, it is rare and since maximum exposure to lifting was also found to be rare, a reduced exposure to heavy lifting would probably not lead to any obvious decrease in the incidence of extremely preterm births in Denmark. 
Still, for legislators and individuals with intensive lifting at work, it is relevant to know that occupational lifting may constitute a risk for preterm birth that could be prevented.

Acknowledgements This study is part of the Danish collaborative MINERVA project addressing occupational risk to human reproduction, which is supported by a grant from the Danish Working Environment Research Fund (contract 20080016458).

Contributors The study was initiated by A-MNA and JPB. The design and analytic strategy were developed by A-MNA, JPB, SWS and SBR. Data were cleaned and analysed by SBR, JKP and MJ. SBR wrote the first version of the manuscript. All authors contributed to the interpretation of the results and the revisions of the manuscript.

Funding The Danish National Research Foundation established the Danish Epidemiology Science Centre that initiated and created the Danish National Birth Cohort (DNBC). The cohort is furthermore a result of a major grant from this foundation. Additional support for the DNBC was obtained from the Pharmacy Foundation, the Egmont Foundation, the March of Dimes Birth Defects Foundation, and the Augustinus Foundation.

\section{Competing interests None.}

Ethics approval DNBC data collection was approved by the Danish Scientific Ethics Committee System and the Danish Data Protection Agency. Approval to use data from the cohort for the present study was obtained from the steering committee of the DNBC.

Provenance and peer review Not commissioned; externally peer reviewed.

\section{REFERENCES}

1 National Collaborating Centre for Women's and Children's Health. Antenatal care: routine care for the healthy pregnant woman. London, UK: RCOG Press, 2008.

2 The Danish Working Environment Authority. WEA Guidelines A1.8. The lay-out of the workplace. The working environment for pregnant and breastfeeding women. (In Danish.) Copenhagen, Denmark: The Danish Working Environment Authority, 2009.

3 The Norwegian Labour Inspection Authority. Pregnancy and working conditions. (In Norwegian.) 2012.

4 Stentebjerg MA, Andersen JH. The influence of physical work conditions on adverse pregnancy outcomes. (In Danish.) Ugeskr Laeger 2006;168:4188-95.

5 National Board of Health. Physical activity - a handbook on prevention and treatment. (In Danish.) Copenhagen, Denmark: National Board of Health, 2003.

6 Directorate for Health and Social Affairs. Guidelines for antenatal care. (In Norwegian.) Oslo, Norway: Directorate for Health and Social Affairs, 2005.

7 Ahlborg G Jr, Bodin L, Hogstedt C. Heavy lifting during pregnancy-a hazard to the fetus? A prospective study. Int I Epidemiol 1990;19:90-7.

8 Bell JF, Zimmerman FJ, Diehr PK. Maternal work and birth outcome disparities. Matern Child Health J 2008;12:415-26.

9 Bonzini M, Coggon D, Palmer KT. Risk of prematurity, low birthweight and pre-eclampsia in relation to working hours and physical activities: a systematic review. Occup Environ Med 2007:64:228-43.
10 Henriksen TB, Hedegaard M, Secher NJ, et al. Standing at work and preterm delivery. Br J Obstet Gynaecol 1995;102:198-206.

11 Misra DP, Strobino DM, Stashinko EE, et al. Effects of physical activity on preterm birth. Am J Epidemiol 1998;147:628-35.

12 Mozurkewich EL, Luke B, Avni M, et al. Working conditions and adverse pregnancy outcome: a meta-analysis. Obstet Gynecol 2000;95:623-35.

13 Pompeii LA, Savitz DA, Evenson KR, et al. Physical exertion at work and the risk of preterm delivery and small-for-gestational-age birth. Obstet Gynecol 2005;106:1279-88.

14 Bonzini M, Coggon D, Godfrey K, et al. Occupational physical activities, working hours and outcome of pregnancy: findings from the Southampton Women's Survey. Occup Environ Med 2009;66:685-90.

15 Burdorf $A$, Brand T, Jaddoe VW, et al. The effects of work-related maternal risk factors on time to pregnancy, preterm birth and birth weight: the Generation $\mathrm{R}$ Study. Occup Environ Med 2011;68:197-204.

16 Goldenberg RL, Culhane JF, lams JD, et al. Epidemiology and causes of preterm birth. Lancet 2008;371:75-84.

17 Wadhwa PD, Culhane JF, Rauh V, et al. Stress, infection and preterm birth: a biobehavioural perspective. Paediatr Perinat Epidemiol 2001;15(Suppl 2):17-29.

18 Lederman RP. Relationship of anxiety, stress, and psychosocial development to reproductive health. Behav Med 1995:21:101-12.

19 Reis FM, Fadalti M, Florio P, et al. Putative role of placental corticotropin-releasing factor in the mechanisms of human parturition. I Soc Gynecol Investig 1999;6:109-19.

20 Sternfeld B. Physical activity and pregnancy outcome. Review and recommendations. Sports Med 1997;23:33-47.

21 Olsen J, Melbye M, Olsen SF, et al. The Danish National Birth Cohort-its background, structure and aim. Scand I Public Health 2001;29:300-7.

22 Bonde JP, Joffe M, Apostoli P, et al. Sperm count and chromatin structure in men exposed to inorganic lead: lowest adverse effect levels. Occup Environ Med 2002;59:234-42

23 Juhl M, Strandberg-Larsen K, Larsen PS, et al. Occupational lifting during pregnancy and risk of fetal death in a large national cohort study. Scand I Work Environ Health 2012 Dec 3. doi: pii: 3335. 10.5271/sjweh.3335. [Epub ahead of print] PubMed PMID: 23207454

24 Madsen $\mathrm{M}$, Jorgensen $\mathrm{T}$, Jensen $\mathrm{ML}$, et al. Leisure time physical exercise during pregnancy and the risk of miscarriage: a study within the Danish National Birth Cohort. BJOG 2007:114:1419-26.

25 Juhl M, Andersen PK, Olsen J, et al. Physical exercise during pregnancy and the risk of preterm birth: a study within the Danish National Birth Cohort. Am J Epidemiol 2008:167:859-66.

26 Langhoff-Roos J, Kesmodel U, Jacobsson B, et al. Spontaneous preterm delivery in primiparous women at low risk in Denmark: population based study. BMJ 2006;332:937-9

27 Nohr EA, Frydenberg $M$, Henriksen $\mathrm{TB}_{\text {, }}$, et al Does low participation in cohort studies induce bias? Epidemiology 2006;17:413-18.

28 Stock SR, Fernandes R, Delisle A, et al. Reproducibility and validity of workers' self-reports of physical work demands. Scand I Work Environ Health 2005;31:409-37.

29 The Danish Ministry of Employment. Analysis of pregnancy related absenteeism. (In Danish.) Copenhagen, Denmark: The Danish Ministry of Employment, 2009. 